\title{
The Ability to Rest at Home During COVID-19 Symptom Manifestation and Depressive Symptoms: Evidence From Korea
}

\author{
Woorim $\mathrm{Kim}^{1 *}$, Yeong Jun $\mathrm{Ju}^{2 \star}$, and Soon Young Lee ${ }^{2 凶}$ \\ ${ }^{1}$ Division of Cancer Control \& Policy, National Cancer Control Institute, National Cancer Center, Goyang, Republic of Korea \\ ${ }^{2}$ Department of Preventive Medicine and Public Health, Ajou University School of Medicine, Suwon, Republic of Korea
}

Objective This study investigated the association between ability to rest at home in times of coronavirus disease 2019 (COVID-19) symptom manifestation and depressive symptoms.

Methods The 2020 Korea Community Health Survey data were used. The presence of depressive symptoms was determined using the Patient Health Questionnaire-9. The relationship between depressive symptoms and ability to rest at home was examined using logistic regression analysis. Additional analysis was performed on the reasons for the inability to stay at home.

Results Depressive symptoms were more common in participants who could not rest at home if symptoms manifested (4.1\%) than those who could rest at home (2.7\%). Participants who could not rest at home (OR 1.53, 95\% CI 1.34-1.74) were more likely to have depressive symptoms, particularly when they had to report to work (OR 1.50, 95\% CI 1.30-1.74) or purchase daily necessities (OR 2.40, 95\% CI 1.15-5.03).

Conclusion Inability to rest at home in the case of COVID-19 symptom manifestation was associated with depressive symptoms. The findings suggest the need to address the identified vulnerable groups to mitigate the mental health consequences of the pandemic.

Psychiatry Investig 2022;19(2):92-99

Keywords COVID-19; Depression; Rest; Symptom.

\section{INTRODUCTION}

The novel coronavirus disease 2019 (COVID-19) has been declared a pandemic by the World Health Organization (WHO) in March 2020. Owing to the rapid spread of the disease worldwide, the number of infected individuals and related deaths is continuously rising drastically. The devastation of the COVID-19 pandemic has inevitably affected the lives and health of numerous people, including various mental health problems. ${ }^{2}$ Increases in the prevalence of psychological disorders during a pandemic are common; studies estimated that up to one-half of the population is vulnerable to experience such disorders. ${ }^{1}$ Likewise, mental health deterioration and depres-

Received: September 8, 2021 Revised: October 28, 2021

Accepted: November 16, 2021

$\triangle$ Correspondence: Soon Young Lee, MD, $\mathrm{PhD}$

Department of Preventive Medicine and Public Health, Ajou University School of Medicine, 206 World cup-ro, Yeongtong-gu, Suwon 16499, Republic of Korea Tel: +82-31-219-5301, Fax: +82-31-219-5084, E-mail: solee@aumc.ac.kr

*These authors contributed equally to this work.

(a) This is an Open Access article distributed under the terms of the Creative Commons Attribution Non-Commercial License (https://creativecommons.org/licenses/by$\mathrm{nc} / 4.0$ ) which permits unrestricted non-commercial use, distribution, and reproduction in any medium, provided the original work is properly cited. sion due to the COVID-19 outbreak have been reported in many countries. ${ }^{3}$ Similar tendencies are shown in South Korea as many residents report higher levels of depression, anxiety, stress, and psychosis risk than before the pandemic. ${ }^{4}$ Moreover, Korea has an exceptionally high suicide rate, which is associated with depression. Therefore, identifying the risk factors for depression and implementing appropriate preventive measures during the pandemic is essential..$^{5}$

Given that exposure to persistent risk and the uncertainty of infectious diseases can escalate risk of depression, the ability to stay at home and rest under times of COVID-19 symptom manifestation may be a relevant factor to investigate. ${ }^{6}$ Psychological responses to pandemic outbreaks may be a result of various factors, including disruptions in daily routines, social isolation, deficiencies in daily necessities, school closures, and occupation related vulnerabilities such as salary deduction. ${ }^{7}$ Vulnerability arising from employment is also a highly correlated factor because COVID-19 related phenomenon, such as the social lockdowns ordered by many governments, have had a substantial impact on the economy and caused escalations in unemployment. ${ }^{8}$ In fact, a large body of literature reveals that involuntary unemployment can have a lasting im- 
pact on mental health, including depression. ${ }^{9}$

As policies on COVID-19 strongly prioritize case isolation and quarantine, requiring symptomatic individuals to undergo home isolation, the ability to rest at home in the case of experiencing disease related symptoms may be associated with risk of depression. ${ }^{10}$ Many countries rely on comprehensive containment policies involving social distancing and the isolation of suspected or confirmed cases whilst vulnerable groups may be more susceptible to being unable to rest despite symptom appearance. ${ }^{11,12}$ Korea has also introduced social distancing and quarantine measures to manage the spread of COVID-19. At the same time, symptoms of acute COVID-19 symptoms have been associated with depressive symptoms, implying the importance of this subject in terms of mental health in times of the pandemic. ${ }^{13}$

This study aimed to investigate the relationship between ability to rest at home if coronavirus symptoms appeared and depressive symptoms, using nationally representative data. We hypothesized that individuals who could not rest at home despite the appearance of suspected coronavirus symptoms were more likely to display depressive symptoms. Additional analysis was conducted to determine the reasons for the inability to rest at home.

\section{METHODS}

\section{Data and study population}

For this study, we used raw data from the 2020 Korea Community Health Survey (KCHS), conducted by the Korea Cen- ters for Disease Control and Prevention. The KCHS is a community-based cross-sectional survey. Sample selection for this survey was conducted using multi-stage stratified probability sampling of civilian and non-institutionalized Korean households. The sample was categorized according to geographic area, age, and sex. The KCHS survey is conducted annually and collects information through in-person (one-on-one) interviews. Because the sample is extracted from national survey data, samples are considered representative of the Korean population. ${ }^{14}$

This study included individuals aged $\geq 19$ years, from an initial total of 229,031 potential participants. The respondents without data on the relevant variables were excluded, leaving 225,744 eligible participants (Figure 1).

\section{Dependent variable}

The dependent variable of this study was depressive symptoms, measured using the Patient Health Questionnaire-9 (PHQ-9). The PHQ-9 is used to screen for depression based on relevant inquiries concerning symptoms experienced in the last two weeks. ${ }^{15}$ The Korean version of the PHQ-9 was previously validated. ${ }^{16}$ Studies on different ethnicities reported that a cut-off score of 10 has a sensitivity of $80 \%$ to $90 \%$ in indicating major depression. ${ }^{17,18}$ Therefore, a score of 10 was also applied in this study to indicate depressive symptoms.

\section{Independent variable}

The independent variable of this study was the individual's ability to rest at home if COVID-19 symptoms appear. This

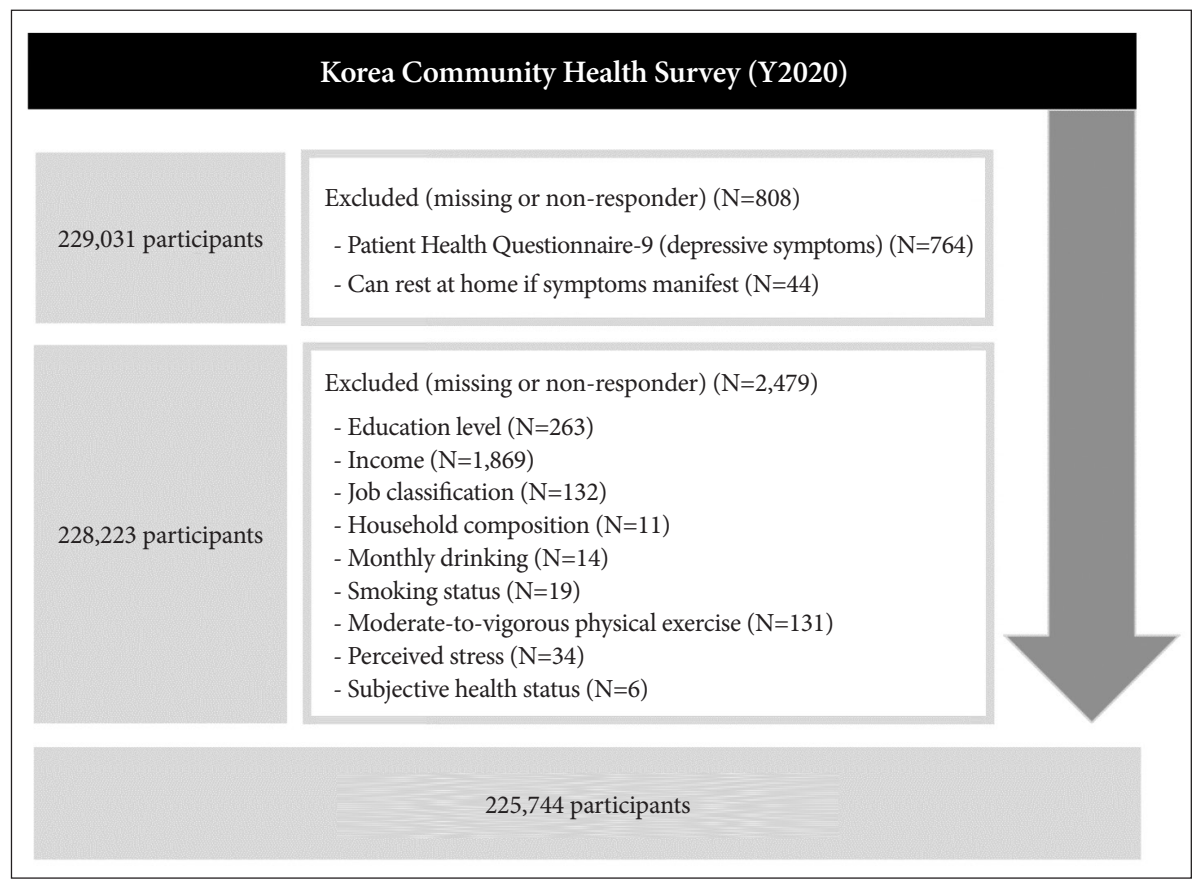

Figure 1. The selection process of the study population. 
Table 1. General characteristics of the study population

\begin{tabular}{|c|c|c|c|c|}
\hline \multirow{2}{*}{ Variables } & \multirow{2}{*}{ Total } & \multicolumn{2}{|c|}{ Depressive symptoms } & \multirow{2}{*}{ p-value } \\
\hline & & No (PHQ-9<10) & Yes (PHQ-9 $\geq 10$ ) & \\
\hline Can rest at home if symptoms manifest & & & & $<0.001$ \\
\hline Yes & $213,864(94.7)$ & $208,003(97.3)$ & $5,861(2.7)$ & \\
\hline No & $11,880(5.3)$ & $11,387(95.9)$ & $493(4.1)$ & \\
\hline Sex & & & & $<0.001$ \\
\hline Male & $102,391(45.4)$ & $100,401(98.1)$ & $1,990(1.9)$ & \\
\hline Female & $123,353(54.6)$ & $118,989(96.5)$ & $4,364(3.5)$ & \\
\hline Age & & & & $<0.001$ \\
\hline $19-29$ & $25,812(11.4)$ & $24,951(96.7)$ & $861(3.3)$ & \\
\hline $30-39$ & $24,985(11.1)$ & $24,231(97.0)$ & $754(3.0)$ & \\
\hline $40-49$ & $35,448(15.7)$ & $34,667(97.8)$ & $781(2.2)$ & \\
\hline $50-59$ & $43,937(19.5)$ & $43,016(97.9)$ & $921(2.1)$ & \\
\hline $60-69$ & $44,380(19.7)$ & $43,394(97.8)$ & $986(2.2)$ & \\
\hline $70+$ & $51,182(22.7)$ & $49,131(96.0)$ & $2,051(4.0)$ & \\
\hline Education & & & & $<0.001$ \\
\hline Uneducated & $20,206(9.0)$ & $19,069(94.4)$ & $1,137(5.6)$ & \\
\hline Elementary school & $32,662(14.5)$ & $31,553(96.6)$ & $1,109(3.4)$ & \\
\hline Middle school & $24,642(10.9)$ & $23,921(97.1)$ & $721(2.9)$ & \\
\hline High school & $77,174(34.2)$ & $75,250(97.5)$ & $1,924(2.5)$ & \\
\hline College and over & $71,060(31.5)$ & $69,597(97.9)$ & $1,463(2.1)$ & \\
\hline Income level & & & & $<0.001$ \\
\hline Q1 & $36,304(16.1)$ & $34,306(94.5)$ & $1,998(5.5)$ & \\
\hline Q2 & $70,996(31.5)$ & $68,907(97.1)$ & $2,089(2.9)$ & \\
\hline Q3 & $55,618(24.6)$ & $54,401(97.8)$ & $1,217(2.2)$ & \\
\hline Q4 & $62,826(27.8)$ & $61,776(98.3)$ & $1,050(1.7)$ & \\
\hline Job classification & & & & $<0.001$ \\
\hline Professional or administrative work & $23,035(10.2)$ & $22,615(98.2)$ & $420(1.8)$ & \\
\hline Office work & $19,684(8.7)$ & $19,317(98.1)$ & $367(1.9)$ & \\
\hline Sales and service & $28,777(12.8)$ & $28,066(97.5)$ & $711(2.5)$ & \\
\hline Agriculture and fishery & $22,213(9.8)$ & $21,906(98.6)$ & $307(1.4)$ & \\
\hline Blue collar work or simple labor & $42,977(19.0)$ & $42,259(98.3)$ & $718(1.7)$ & \\
\hline Others (unemployed, student, etc.) & $89,058(39.5)$ & $85,227(95.7)$ & $3,831(4.3)$ & \\
\hline Household composition & & & & $<0.001$ \\
\hline 1 generation & $107,369(47.6)$ & $104,014(96.9)$ & $3,355(3.1)$ & \\
\hline 2 generation & $103,538(45.9)$ & $100,944(97.5)$ & $2,594(2.5)$ & \\
\hline 3 generation & $14,837(6.6)$ & $14,432(97.3)$ & $405(2.7)$ & \\
\hline Area of residence & & & & $<0.001$ \\
\hline Rural & $98,671(43.7)$ & $96,143(97.4)$ & $2,528(2.6)$ & \\
\hline Urban & $127,073(56.3)$ & $123,247(97.0)$ & $3,826(3.0)$ & \\
\hline Monthly drinking status & & & & $<0.001$ \\
\hline No & $124,410(55.1)$ & $120,383(96.8)$ & $4,027(3.2)$ & \\
\hline Yes & $101,334(44.9)$ & $99,007(97.7)$ & $2,327(2.3)$ & \\
\hline Smoking status & & & & $<0.001$ \\
\hline No & $189,244(83.8)$ & $184,108(97.3)$ & $5,136(2.7)$ & \\
\hline Yes & $36,500(16.2)$ & $35,282(96.7)$ & $1,218(3.3)$ & \\
\hline
\end{tabular}


Table 1. General characteristics of the study population (continued)

\begin{tabular}{|c|c|c|c|c|}
\hline \multirow{2}{*}{ Variables } & \multirow{2}{*}{ Total } & \multicolumn{2}{|c|}{ Depressive symptoms } & \multirow{2}{*}{$\mathrm{p}$-value } \\
\hline & & No (PHQ-9<10) & Yes (PHQ-9 $\geq 10)$ & \\
\hline Moderate-to-vigorous physical exercise & & & & $<0.001$ \\
\hline No & $182,106(80.7)$ & $176,639(97.0)$ & $5,467(3.0)$ & \\
\hline Yes & $43,638(19.3)$ & $42,751(98.0)$ & $887(2.0)$ & \\
\hline Perceived stress & & & & $<0.001$ \\
\hline No & $175,845(77.9)$ & $174,078(99.0)$ & $1,767(1.0)$ & \\
\hline Yes & $49,899(22.1)$ & $45,312(90.8)$ & $4,587(9.2)$ & \\
\hline Subjective health status & & & & $<0.001$ \\
\hline Poor & $117,285(52.0)$ & $111,950(95.5)$ & $5,335(4.6)$ & \\
\hline Fair & $108,459(48.1)$ & $107,440(99.1)$ & $1,019(0.9)$ & \\
\hline Total & $225,744(100.0)$ & $219,390(97.2)$ & $6,354(2.8)$ & \\
\hline
\end{tabular}

Data are presented as N (\%). PHQ-9, Patient Health Questionnaire-9

was measured based on the following question: "Are you able to stay at home and rest if you have COVID-19 related symptoms, such as a fever or respiratory symptoms?" Available responses were 'yes' and 'no.' Participants who responded 'no' to this question were asked a follow-up question about the main reasons for their inability to rest at home. Available answers for the follow up question were 1) because of work, 2) because of the need to purchase daily necessities such as food and medicine, 3) because of the need to care for family members or friends, and 4) For other reasons. The reasons for the inability to work were further analyzed during this study.

\section{Covariates}

Various covariates were accounted for in the analysis. The included covariates were sex (male or female), age (19 to 29, 30 to 39,40 to 49,50 to 59,60 to 69 , or 70 or above), education (uneducated, elementary school, middle school, high school, college or above), income level (quartiles), job classification (professional or administrative work, office work, sales and service, agriculture and fishery, blue-collar work or simple labor, or others [unemployed, and students]), household composition (one, two, or three-generation household), area or residence (rural or urban), monthly drinking status (no or yes), smoking status (no or yes), moderate to vigorous physical exercise (no or yes), perceived stress (no or yes), and subjective health status (poor or fair). Area of residence was classified into urban or rural based on data collected directly by the KCHS as the KCHS uses the region variable for stratification. Current smokers included current or occasional smokers who responded to have smoked at least 100 cigarettes (five packs) or more during their life time. Monthly drinkers included individuals who currently drink at least once a month and responded to have drank at least one shot of alcohol during their lifetime.

\section{Statistical analysis}

The general characteristics were examined using the chisquare test to identify significant differences between groups. Multivariable logistic regression analysis was then used to examine the association between ability to rest at home if COVID-19 symptoms manifested and depressive symptoms while controlling for potential confounding variables. These variables include sex, age, education, income, occupation, household composition, area of residence, alcohol consumption in the preceding month, smoking status, physical exercise, perceived stress, and subjective health status. Subgroup analysis was performed to evaluate possible associations between perceived social support and depressive symptoms in terms of income. Income was selected because previous studies report that disadvantaged individuals may share an unequal burden resulting from the outbreak, with low income being associated with higher risk of COVID-19 mortality. Results were expressed as odds ratio (OR) at 95\% confidence interval (CI). All analyses were conducted using SAS 9.4 (SAS Institute, Cary, NC, USA) software. p-values were two-sided and considered significant at $\mathrm{p}<0.05$.

\section{Ethical approval}

The authors assert that all procedures contributing to this work comply with the ethical standards of the relevant national and institutional committees on human experimentation and with the Helsinki Declaration of 1975, as revised in 2008. In addition, the Korea Community Health Survey (KCHS) data are openly published. Participants' data were fully anonymized prior to release. Our study was excluded from the review list pursuant to Article 2.2 of the Enforcement Rule of Bioethics and Safety Act in Korea, since the data was exempted from IRB review. 
Table 2. The association between depressive symptoms and being able to rest at home in the case of COVID-19 symptom manifestation

\begin{tabular}{ccc}
\hline \multirow{2}{*}{ Variables } & \multicolumn{2}{c}{ Depressive symptoms } \\
\cline { 2 - 3 } & Adjusted-OR & $95 \% \mathrm{CI}$ \\
\hline
\end{tabular}

Can rest at home if symptoms manifest

Yes

No

Sex

Male

Age

19-29

$30-39$

40-49

$50-59$

60-69

$70+$

Education

Uneducated

Elementary school

Middle school

High school

College and over

Income level

Q1

Q2

Q3

Q4

Job classification

Professional or administrative

work

Office work

Sales and service

Agriculture and fishery

Blue collar work or simple labor

Others (unemployed, student, etc.)

Household composition

1 generation

1.00

2 generation

0.92

1.11

$0.85-1.01$

3 generation

Area of residence

Rural

1.00

Urban

1.14

$1.04-1.25$

Monthly drinking status

\begin{tabular}{lll} 
No & 1.00 & \\
Yes & 1.00 & $0.92-1.09$ \\
\hline
\end{tabular}

Table 2. The association between depressive symptoms and being able to rest at home in the case of COVID-19 symptom manifestation (continued)

\begin{tabular}{lcc}
\hline \multirow{2}{*}{ Variables } & \multicolumn{2}{c}{ Depressive symptoms } \\
\cline { 2 - 3 } & Adjusted-OR & $95 \% \mathrm{CI}$ \\
\hline Smoking status & & \\
$\quad$ No & 1.00 & \\
Yes & 1.63 & $1.47-1.82$ \\
Moderate-to-vigorous physical exercise & & \\
$\quad$ No & 1.00 & \\
$\quad$ Yes & 0.99 & $0.90-1.10$ \\
Perceived stress & & \\
$\quad$ No & 1.00 & \\
$\quad$ Yes & 9.57 & $8.82-10.38$ \\
Subjective health status & & \\
$\quad$ Poor & 1.00 & \\
Fair & 3.25 & $2.96-3.56$ \\
\hline OR, odds ratio; CI, confidence interval & &
\end{tabular}

\section{RESULTS}

Table 1 presents the general characteristics of the study's participants. Of the 225,744 participants, 6,354 (2.8\%) had depressive symptoms. Depressive symptoms were more common in participants who responded that they could not rest at home (4.1\%) when experiencing COVID-19-related symptoms than those who could (2.7\%). Depressive symptoms were also more prevalent in lower-income groups than in higherincome groups. The percentage of participants with depressive symptoms decreased in a stepwise manner with each income level group. With regard to individuals who could not rest at home, the population distribution for each reason behind not being able to rest can be found in Supplementary Table 1(in the online-only Data Supplement).

Table 2 reveals the association between depressive symptoms and ability to rest at home if COVID-19 symptoms appeared. Compared to individuals who could rest, those who could not (OR 1.53, 95\% CI 1.34-1.74) were more likely to have depressive symptoms. The additional analysis on the reasons for not resting at home is shown in Table 3. Higher odds of depressive symptoms were found in participants who could not rest because of work (OR 1.50, 95\% CI 1.30-1.74), the need to purchase daily necessities (OR 2.40, 95\% CI 1.155.03), and other reasons (OR 1.64, 95\% CI 1.19-2.24), than those who could rest at home if they experienced symptoms.

Table 4 shows the results of the subgroup analysis conducted based on income level. The general tendencies of the main findings were maintained regardless of income level, which infers that the inability to rest at home in case of the emergence 
Table 3. The association between depressive symptoms and the reasons behind not being able to rest at home

\begin{tabular}{|c|c|c|}
\hline \multirow{2}{*}{ Variables } & \multicolumn{2}{|c|}{ Depressive symptoms } \\
\hline & Adjusted-OR & $95 \% \mathrm{CI}$ \\
\hline \multicolumn{3}{|l|}{ Reasons behind not being able to rest at home } \\
\hline Can rest at home & 1.00 & \\
\hline Because of work & 1.50 & $1.30-1.74$ \\
\hline Because of the need to purchase daily necessities & 2.40 & $1.15-5.03$ \\
\hline Because of the need to take care of family members or friends & 1.29 & $0.73-2.28$ \\
\hline Others & 1.64 & $1.19-2.24$ \\
\hline
\end{tabular}

Adjusted for sex, age, education income level, job classification, household composition, area of residence, monthly drinking status, smoking status, physical exercise, perceived stress, and subjective health status. OR, odds ratio; CI, confidence interval

Table 4. The results of the subgroup analysis

\begin{tabular}{clcc}
\hline & \multirow{2}{*}{ Variables } & \multicolumn{2}{c}{ Depressive symptoms } \\
\cline { 3 - 4 } Income level & Can rest at home & Adjusted-OR & 95\% CI \\
Low & Yes & 1.00 & \\
\multirow{4}{*}{ Middle-low } & No & 2.03 & $1.35-3.05$ \\
& Yes & 1.00 & \\
Middle-high & No & 1.39 & $1.10-1.75$ \\
& Yes & 1.00 & \\
High & No & 1.56 & $1.24-1.96$ \\
& Yes & 1.00 & \\
\hline & No & 1.58 & $1.24-2.01$ \\
\hline
\end{tabular}

Adjusted for sex, age, education income level, job classification, household composition, area of residence, monthly drinking status, smoking status, physical exercise, perceived stress, and subjective health status. OR, odds ratio; CI, confidence interval

of COVID-19 symptoms is related to significantly higher odds of depressive symptoms in all income groups. However, the magnitude of this association was relatively higher among participants belonging to the low (OR 2.03, 95\% CI 1.35-3.05) than the middle-low (OR 1.39, 95\% CI 1.10-1.75), middlehigh (OR 1.56, 95\% CI 1.24-1.96), and high (OR 1.58, 95\% CI 1.24-2.01) income groups.

\section{DISCUSSION}

This study is the first to establish an association between the inability to rest at home if COVID-19-related symptoms manifested and increased depressive symptoms, using nationally representative data from Korea. The study's findings revealed that depressive symptoms were more likely in participants who could not rest at home due to work or the need to purchase daily necessities. The association between depressive symptoms and ability to rest at home if COVID-19 symptoms emerge during the pandemic was significant in all income groups-the magnitude of this relationship being strongest in the low-income group. The study results offer important insights, because many countries have adopted COVID-19 containment policies focusing on social distancing and suspected or confirmed case isolation. However, studies showed that lasting pandemics increase the general population's vulnerability to depressive symptoms. ${ }^{19}$ The analyses of this study demonstrated that addressing potentially disadvantaged individuals who cannot rest at home despite suspicion of COVID-19 infection may be necessary in managing and mitigating depression during times of infectious disease outbreaks.

The reasons for the inability to rest at home, even while experiencing COVID-19 symptoms, can be diverse. These reasons included work-related responsibilities, the need to purchase daily necessities, and the need to care for family members or friends. Based on this study's result, it can be concluded that the inability to stay at home because of work or having to purchase daily necessities was related to depressive symptoms. Regarding work-related reasons, occupational insecurity and financial concerns due to the COVID-19 outbreak have been associated with greater depressive symptoms. ${ }^{20}$ Specifically, individuals with perceived job insecurity had a higher likelihood of depressive symptoms. Additionally, individuals with financial stress reported a twofold increase in the risk of showing clinically depressive symptoms. ${ }^{21}$ In contrast, having a job to return to and the ability to take paid leave during the outbreak had significant mental health benefits. ${ }^{8}$ Currently, the Korean government requires individuals to self-monitor for COVID-19 symptoms, isolate themselves, and get tested upon symptom development. Under such circumstances, being unable to stay at home due to work may suggest job insecurity or experiences of financial constraints, which can increase the risk of depressive symptoms.

Likewise, individuals needing to purchase daily commodities during the outbreak may be from a relatively disadvantaged group, such as adults living alone. This is an important factor to investigate because a previous survey revealed that the most common reason to go outside despite being requested to stay at home during the COVID-19 outbreak was to buy 
food..$^{22}$ Studies have shown that person-to-person contact is essential for maintaining positive emotions, and living alone can increase the risk for poor well-being. ${ }^{23}$ Marital status has also been reported to correlate with psychological distress, with married people showing lower levels of distress than single individuals during the pandemic. ${ }^{24}$ Additionally, social isolation may escalate the risk of food insecurity and nutritional insufficiency, which play a significant role in the psychological health of older adults. ${ }^{25}$ Based on these results, there is a need to address the identified group of individuals who may be vulnerable to depressive symptoms during the pandemic.

The relationship between the inability to rest at home and depressive symptoms during the COVID-19 outbreak was maintained regardless of income level, with the strongest degree of correlation being present in the lowest income group. This result suggests that ability to stay at home in case COVID-19 symptoms appeared is an influential factor for depressive symptoms, which merits attention because of the extent of the resulting psychological distress. ${ }^{4}$ Acute COVID-19 symptoms are also known to have a bidirectional relationship with depressive symptoms, emphasizing the importance of this factor in addressing mental health during the pandemic. ${ }^{13}$ Moreover, further emphasis should be placed on lower-income groups because the socioeconomically disadvantaged are likely to experience disproportionately higher risks of mental illnesses when disruptive, traumatic events occur. ${ }^{26}$

This study has several limitations. First, causal relationships cannot be confirmed because the data used were cross-sectional. Second, the interesting variable of this study was measured based on self-reports only. Additionally, some participants did not provide specific reasons for their inability to remain at home; instead, they chose the 'other reasons' option. Third, respondents may have participated at different peak times of the COVID-19 pandemic as the number of infected patients continuously fluctuated. Last, the fact that reasons for not being able to stay at home during the pandemic may differ depending on age needs to be considered when interpreting the study results. ${ }^{27}$ Despite the limitations stated above, this study is important because it is the first to investigate the relationship between the inability to rest at home in the case of COVID-19 symptom appearance and depressive symptoms. The findings are also significant because the data consisted of a large, nationally representative sample of the general population surveyed during the COVID-19 crisis.

In conclusion, inability to rest at home in the case of COVID-19 symptom manifestation was associated with higher depressive symptoms. Specifically, an increased likelihood of depressive symptoms was found in participants who reported work-related responsibilities or the need to purchase daily necessities as reasons for their inability to stay home. The ten- dencies of the general findings were maintained regardless of income level, with the lowest income group showing the most significant degree of association. The findings suggest the need to address the identified vulnerable group of individuals in mitigating the mental health consequences of the pandemic.

\section{Supplementary Materials}

The online-only Data Supplement is available with this article at https://doi.org/10.30773/pi.2021.0300.

\section{Availability of Data and Material}

Data will be made available on request. The dataset is available on the Korea Community Health Survey website (https://chs.cdc.go.kr/chs/rdr/ rdrInfoProcessMain.do).

\section{Conflicts of Interest}

The authors have no potential conflicts of interest to disclose.

\section{Author Contributions}

Conceptualization: Yeong Jun Ju. Data curation: Woorim Kim, Yeong Jun Ju. Formal analysis: Yeong Jun Ju. Investigation: Woorim Kim, Yeong Jun Ju. Project administration: Soon Young Lee. Supervision: Soon Young Lee. Validation: Soon Young Lee. Writing-original draft: Woorim Kim. Writing—review \& editing: Woorim Kim.

\section{ORCID iDs}

Woorim Kim https://orcid.org/0000-0002-1199-6822

Yeong Jun Ju https://orcid.org/0000-0003-4891-0524

Soon Young Lee https://orcid.org/0000-0002-3160-577X

\section{Funding Statement \\ None}

\section{REFERENCES}

1. Khademian F, Delavari S, Koohjani Z, Khademian Z. An investigation of depression, anxiety, and stress and its relating factors during COVID-19 pandemic in Iran. BMC Public Health 2021;21:275.

2. Shigemura J, Ursano RJ, Morganstein JC, Kurosawa M, Benedek DM. Public responses to the novel 2019 coronavirus (2019-nCoV) in Japan: Mental health consequences and target populations. Psychiatry Clin Neurosci 2020;74:281-282.

3. Bueno-Notivol J, Gracia-Garcia P, Olaya B, Lasheras I, Lopez-Anton R, Santabarbara J. Prevalence of depression during the COVID-19 outbreak: a meta-analysis of community-based studies. Int J Clin Health Psychol 2021;21:100196.

4. Lee HS, Dean D, Baxter T, Griffith T, Park S. Deterioration of mental health despite successful control of the COVID-19 pandemic in South Korea. Psychiatry Res 2021;295:113570.

5. Organization for Economic Development and Cooperation (OECD). Health at a Glance 2019: OECD Indicators. Paris: OECD Publishing; 2019.

6. Qi M, Zhou SJ, Guo ZC, Zhang LG, Min HJ, Li XM, et al. The effect of social support on mental health in Chinese adolescents during the outbreak of COVID-19. J Adolesc Health 2020;67:514-518.

7. Choi EPH, Hui BPH, Wan EYF. Depression and Anxiety in Hong Kong during COVID-19. Int J Environ Res Public Health 2020;17:3740.

8. Posel D, Oyenubi A, Kollamparambil U. Job loss and mental health during the COVID-19 lockdown: Evidence from South Africa. PLoS One 2021;16:e0249352.

9. Chapman B, Swainston J, Grunfeld EA, Derakshan N. COVID-19 outbreak effects on job security and emotional functioning amongst wom- 
en living with breast cancer. Front Psychol 2020;11:582014.

10. Guan WJ, Ni ZY, Hu Y, Liang WH, Ou CQ, He JX, et al. Clinical characteristics of coronavirus disease 2019 in China. N Engl J Med 2020;382: 1708-1720.

11. Dighe A, Cattarino L, Cuomo-Dannenburg G, Skarp J, Imai N, Bhatia $S$, et al. Response to COVID-19 in South Korea and implications for lifting stringent interventions. BMC Med 2020;18:321.

12. Nagasu M, Muto K, Yamamoto I. Impacts of anxiety and socioeconomic factors on mental health in the early phases of the COVID-19 pandemic in the general population in Japan: a web-based survey. PLoS One 2021; 16:e0247705.

13. Perlis RH, Ognyanova K, Santillana M, Baum MA, Lazer D, Druckman J, et al. Association of acute symptoms of COVID-19 and symptoms of depression in adults. JAMA Netw Open 2021;4:e213223.

14. Kang YW, Ko YS, Kim YJ, Sung KM, Kim HJ, Choi HY, et al. Korea community health survey data profiles. Osong Public Health Res Perspect 2015;6:211-217.

15. Shin C, Kim Y, Park S, Yoon S, Ko YH, Kim YK, et al. Prevalence and associated factors of depression in general population of Korea: results from the Korea National Health and Nutrition Examination Survey, 2014. J Korean Med Sci 2017;32:1861-1869.

16. Han C, Jo SA, Kwak JH, Pae CU, Steffens D, Jo I, et al. Validation of the atient Health Questionnaire-9 Korean version in the elderly population: the Ansan Geriatric study. Compr Psychiatry 2008;49:218-223.

17. Kroenke K, Spitzer RL, Williams JB. The PHQ-9: validity of a brief depression severity measure. J Gen Intern Med 2001;16:606-613.

18. Levis B, Benedetti A, Thombs BD; Depression Screening Data (DEPRESSD) Collaboration. Accuracy of Patient Health Questionnaire-9 (PHQ-9) for screening to detect major depression: individual participant data meta-analysis. BMJ 2019;365:11476.

19. Ettman CK, Abdalla SM, Cohen GH, Sampson L, Vivier PM, Galea S. Prevalence of depression symptoms in US adults before and during the
COVID-19 pandemic. JAMA Network Open 2020;3:e2019686-e2019686.

20. Wilson JM, Lee J, Fitzgerald HN, Oosterhoff B, Sevi B, Shook NJ. Job insecurity and financial concern during the COVID-19 pandemic are associated with worse mental health. J Occup Environ Med 2020;62: 686-691.

21. Ruengorn C, Awiphan R, Wongpakaran N, Wongpakaran T, Nochaiwong $\mathrm{S}$, Health $\mathrm{O}$, et al. Association of job loss, income loss, and financial burden with adverse mental health outcomes during coronavirus disease 2019 pandemic in Thailand: a nationwide cross-sectional study. Depress Anxiety 2021;38:648-660.

22. Ozdin S, Bayrak Ozdin S. Levels and predictors of anxiety, depression and health anxiety during COVID-19 pandemic in Turkish society: the importance of gender. Int J Soc Psychiatry 2020;66:504-511.

23. Fingerman KL, Ng YT, Zhang S, Britt K, Colera G, Birditt KS, et al. Living Alone During COVID-19: Social Contact and Emotional Well-being Among Older Adults. J Gerontol B Psychol Sci Soc Sci 2021;76:e116e121.

24. Wang H, Xia Q, Xiong Z, Li Z, Xiang W, Yuan Y, et al. The psychological distress and coping styles in the early stages of the 2019 coronavirus disease (COVID-19) epidemic in the general mainland Chinese population: a web-based survey. PLoS One 2020;15:e0233410.

25. Malek Rivan NF, Yahya HM, Shahar S, Ajit Singh DK, Ibrahim N, Mat Ludin AF, et al. The impact of poor nutrient intakes and food insecurity on the psychological distress among community-dwelling middleaged and older adults during the COVID-19 pandemic. Nutrients 2021; 13:353.

26. Tracy M, Norris FH, Galea S. Differences in the determinants of posttraumatic stress disorder and depression after a mass traumatic event. Depress Anxiety 2011;28:666-675.

27. Drefahl S, Wallace M, Mussino E, Aradhya S, Kolk M, Branden M, et al. A population-based cohort study of socio-demographic risk factors for COVID-19 deaths in Sweden. Nat Commun 2020;11:5097. 
Supplementary Table 1. The distribution of each reason for behind not being able to rest at home

\begin{tabular}{lrrr}
\hline \multicolumn{1}{c}{ Variables } & Total & \multicolumn{2}{c}{ Depressive symptoms } \\
& & No (PHQ-9<10) & Yes (PHQ-9 $\geq 10)$ \\
\hline Reasons behind not being able to rest at home & & & \\
Can rest at home & $213,864(94.7)$ & $208,003(97.3)$ & $5,861(2.7)$ \\
Because of work & $10,077(4.5)$ & $9,701(96.3)$ & $376(3.7)$ \\
Because of the need to purchase daily necessities & $214(0.1)$ & $200(93.5)$ & $14(6.5)$ \\
Because of the need to take care of family members or friends & $322(0.1)$ & $302(93.8)$ & $20(6.2)$ \\
Others & $1,267(0.6)$ & $1,184(93.4)$ & $83(6.6)$ \\
\hline
\end{tabular}

Data are presented as N (\%). PHQ-9, Patient Health Questionnaire-9 